\title{
Judicious partitions and related problems
}

\author{
Alex Scott
}

\begin{abstract}
Many classical partitioning problems in combinatorics ask for a single quantity to be maximized or minimized over a set of partitions of a combinatorial object. For instance, Max Cut asks for the largest bipartite subgraph of a graph $G$, while Min Bisection asks for the minimum size of a cut into two equal pieces.

In judicious partitioning problems, we seek to maximize or minimize a number of quantities simultaneously. For instance, given a graph $G$ with $m$ edges, we can ask for the smallest $f(m)$ such that $G$ must have a bipartition in which each vertex class contains at most $f(m)$ edges.

In this survey, we discuss recent extremal results on a variety of questions concerning judicious partitions, and related problems such as Max Cut.
\end{abstract}

\section{Introduction}

A wide variety of combinatorial optimization problems ask for an "optimal" partition of the vertex set of a graph or hypergraph. A good example is the Max Cut problem: given a graph $G$, what is the maximum of $e\left(V_{1}, V_{2}\right)$ over partitions $V(G)=V_{1} \cup V_{2}$, where $e\left(V_{1}, V_{2}\right)$ is the number of edges between $V_{1}$ and $V_{2}$ ? Similarly, Min Bisection asks for the minimum of $e\left(V_{1}, V_{2}\right)$ over partitions $V(G)=V_{1} \cup V_{2}$ with $\left|V_{1}\right| \leq\left|V_{2}\right| \leq$ $\left|V_{1}\right|+1$ (there are $k$-partite versions Max $k$-Cut and Min $k$-Section of both problems).

Both of these problems involve maximizing or minimizing a single quantity over graphs from a certain class. In this survey, we shall discuss a group of problems where several quantities must be maximized or minimized simultaneously. There are a number of variations, but we will group these problems together under the heading of judicious partitioning problems.

For example, given a graph $G$, what is the minimum of $\max \left\{e\left(V_{1}\right), e\left(V_{2}\right)\right\}$ over partitions $V(G)=V_{1} \cup V_{2}$ ? Note the difference here from Max Cut: in Max Cut, we are looking for a partition in which $e\left(V_{1}, V_{2}\right)$ is large, or equivalently $e\left(V_{1}\right)+e\left(V_{2}\right)$ is small, but we do not care how edges are shared between $V_{1}$ and $V_{2}$. Here we are seeking a partition in which $e\left(V_{1}\right)$ and $e\left(V_{2}\right)$ are small simultaneously.

In general, judicious partitioning problems seem to be more difficult than similar partitioning problems in which a single quantity is optimized. For instance, in the case of Max Cut it is easy to show that every graph with $m$ edges has a bipartite subgraph with at least $m / 2$ edges: a random bipartition, in which each vertex is independently assigned to either class with equal probability, gives a bipartite subgraph with expected size $m / 2$, and so there must be some bipartite subgraph of at least this size (and random graphs show that the constant $1 / 2$ is best possible). But what if we want a judicious bipartition, in which both vertex classes contain few edges? In a random bipartition $V(G)=V_{1} \cup V_{2}$ of a graph with $m$ edges, we expect $m / 4$ edges in each vertex class; but this does not imply the existence of a good judicious partition, as the quantities $e\left(V_{1}\right)$ and $e\left(V_{2}\right)$ are not independent (for instance, a random bipartition of $K_{1, n-1}$ has, with high probability, about $n / 2$ edges on one side and no edges on the other side). Thus we cannot expect to get good bounds for this judicious partitioning problem from a naive random argument. 
Indeed, it is far from easy to determine the largest $c$ such that every graph with $m$ edges has a bipartition with at most $\mathrm{cm}$ edges in each vertex class. A similar problem holds in the algorithmic context: for Max Cut, a simple greedy algorithm finds a cut of size at least $m / 2$ in linear $(O(m+n))$ time; finding a reasonably good judicious partition appears to be rather more complicated.

In general, partitioning problems have two aspects: an extremal problem and an algorithmic problem. The extremal problem asks for bounds on the size of a largest cut. For instance, given $m \geq 1$, what is the largest $m^{\prime}$ such that every graph with $m$ edges has a cut of size at least $m^{\prime}$ ? The algorithmic problem asks for efficient algorithms (or heuristics) to find large cuts, or else a proof that it is hard (in some appropriate sense) to find a large cut, or to determine whether one exists. We shall focus here on extremal problems, although these are often closely related to algorithmic questions: extremal results often give structural information that can be used to design efficient algorithms, or explain why algorithms perform well or badly.

Judicious partitioning problems arguably share certain features with many realworld problems, where multiple constraints and objectives must be taken into account (for example, many measures of success in circuit layout problems can be thought of as judicious partitioning problems; see [33]). However, real-world problems are often sufficiently complex and specific that their mathematical structure is obscured. The problems considered in this paper are hopefully complex enough to exhibit interesting behaviour, but simple enough to remain mathematically interesting. Even with very simple judicious partitioning problems, many fascinating open questions remain.

The remainder of the paper is split into three sections. In the first two sections, we consider partitioning problems on graphs: we begin with problems with a single constraint, concentrating in particular on Max Cut and its variants, while in section 3 , we move on to discuss a variety of judicious partitioning problems. In the final section, we look at partitioning problems on hypergraphs: these are in general much more difficult than the corresponding graph partitioning problems, but some progress has been made, and there are some tantalising conjectures.

A large part of this survey is based on a series of joint papers with Béla Bollobás $[9,10,11,12,13,14,15,16,17,18]$, and much of the remainder is influenced by joint discussions. Any mistakes, of course, are mine.

\subsection{Notation}

For a graph $G$ and $X \subset V(G)$, we define $G[X]$ to be the subgraph induced by $X$ and set $e(X)=e(G[X])$. For disjoint subsets $X, Y \subset V(G)$, we write

$$
e(X, Y)=|\{x y \in E(G): x \in X, y \in Y\}|
$$

for the number of edges between $X$ and $Y$. If $G$ is a digraph, $e(X, Y)$ denotes the number of edges that are directed from $X$ to $Y$.

If $G$ is a graph with vertex set $V$, then a cut $\left(V_{1}, V_{2}\right)$ of $G$ is a bipartition $V=V_{1} \cup V_{2}$; a bisection of $G$ is a cut with $\left|V_{1}\right| \leq\left|V_{2}\right| \leq\left|V_{1}\right|+1$. The size of the cut $\left(V_{1}, V_{2}\right)$ is $e\left(V_{1}, V_{2}\right)$. Similarly, if $G$ is a digraph, a directed cut of $G$ is an ordered pair $\left(V_{1}, V_{2}\right)$ such that $V_{1} \cup V_{2}$ partitions $V(G)$; the size of the directed cut is $e\left(V_{1}, V_{2}\right)$ (i.e. we only count edges from $V_{1}$ to $\left.V_{2}\right)$. 


\section{Graph partitions with a single objective}

\subsection{Max Cut}

Probably the best known graph partitioning problem is Max Cut, which asks for the largest bipartite subgraph of a graph $G$. The extremal version of the problem asks for bounds on the size of a maximum cut in terms of various parameters of $G$; the algorithmic version, which has an immense literature, asks for algorithms to find a maximum cut or to determine its size, or for proofs that it is in some sense hard to do so. Important surveys of the Max Cut problem can be found in Poljak and Tuza [38] and Laurent [31].

We concentrate here on the extremal version of the problem. For a graph $G$, we define

$$
f(G)=\max _{W \subset V} e(W, V \backslash W)
$$

to be the maximal size of a bipartite subgraph of $G$. For $m \geq 1$, we write

$$
f(m)=\min _{e(G)=m} f(G) .
$$

We will also consider Max Cut for edge-weighted graphs. For a graph $G$ with edgeweighting $w$, we define

$$
f(G)=\max _{W \subset V} w(W, V \backslash W),
$$

where $w(W, V \backslash W)=\sum_{u \in W, v \in V \backslash W} w(u v)$ is the weight of the cut $(W, V \backslash W)$. We further define

$$
f_{w}(m)=\min _{w(G)=m} f(G),
$$

where the minimum is taken over all graphs whose edges are weighted with positive integers and have total weight $m$. Equivalently, $f_{w}(m)$ is the minimum value of $f(G)$ over multigraphs with $m$ edges. Clearly

$$
f_{w}(m) \leq f(m) .
$$

It is easily seen (for instance, by considering random bipartitions) that

$$
f_{w}(m) \geq m / 2 \text {. }
$$

In 1973, answering a question of Erdős, Edwards [22, 21] proved that

$$
f(m) \geq \frac{m}{2}+\sqrt{\frac{m}{8}+\frac{1}{64}}-\frac{1}{8} .
$$

The extremal graphs are the complete graphs of odd order.

We will give two proofs of (2.2). For a first proof, we note that (2.2) follows immediately from (2.1) and the following theorem.

Theorem 2.1 For every $m$,

$$
f_{w}(m) \geq \frac{m}{2}+\sqrt{\frac{m}{8}+\frac{1}{64}}-\frac{1}{8} .
$$


We give a short proof of Theorem 2.1 due to Alon [3] and Hofmeister and Lefmann [28].

Proof Let $G$ be a graph with $w(G)=m$ and $f_{w}(G)$ minimal. If $G$ contains two vertices $x$ and $y$ that are not adjacent (or for which $w(x y)=0$ ), we can compress $G$ by identifying $x$ and $y$ to create a new vertex $z$. We define

$$
w(z a)=w(x a)+w(y a)
$$

for all $a \in V(G) \backslash\{x, y\}$, and leave other edge-weights unchanged (we define $w(u v)=$ 0 if $u v$ is not an edge). Let $H$ be the resulting graph. Any bipartition of $H$ can be extended to a bipartition of $G$ with the same weight by replacing $z$ with $x$ and $y$, so $f(H) \leq f(G)$. By repeated compressions, we may therefore assume that $G$ is an edge-weighting of the complete graph.

Now let $n=|G|$, and consider a bipartition chosen uniformly at random from all bipartitions of $V(G)$ into sets of size $\lfloor n / 2\rfloor$ and $\lceil n / 2\rceil$. Let's check that this has expected weight at least (2.3). The expected weight of our bipartition is

$$
\frac{\left\lfloor n^{2} / 4\right\rfloor}{\left(\begin{array}{c}
n \\
2
\end{array}\right)} m \geq \frac{\left(n^{2}-1\right) / 4}{n(n-1) / 2} m=\frac{n+1}{2 n} m .
$$

Defining $n^{\prime}$ by $m=\left(\begin{array}{c}n^{\prime} \\ 2\end{array}\right)$, we see that this is at least

$$
\begin{aligned}
\frac{m}{2}+\frac{m}{2 n} & \geq \frac{m}{2}+\frac{m}{2 n^{\prime}} \\
& =\frac{m}{2}+\frac{n^{\prime}-1}{4} \\
& =\frac{m}{2}+\sqrt{\frac{m}{8}+\frac{1}{64}}-\frac{1}{8}
\end{aligned}
$$

where the last equality follows from the fact that $m=\left(\begin{array}{c}n^{\prime} \\ 2\end{array}\right)$.

A similar idea (in this case, contracting colour classes to a single vertex) gives the following simple bound observed by several authors $[3,6,32,34]$.

Theorem 2.2 For a nonempty graph $G$ with $m$ edges,

$$
f(G) \geq\left(\frac{1}{2}+\frac{1}{2 \chi(G)}\right) m
$$

Another useful bound on $f(G)$ was proved by Edwards [22].

Lemma 2.3 If $G$ is a connected graph then

$$
f(G) \geq \frac{e(G)}{2}+\frac{|G|-1}{4} .
$$

Short proofs or algorithms can be found in [26, 28, 37, 36, 14]. Note that the bound is sharp for complete graphs of odd order. 
Lemma 2.3 also gives a short second proof of (2.2). Identifying a vertex from each component, we may assume that $G$ is connected; if $G$ has $n$ vertices and $m$ edges then it is easily checked (as in the proof of Lemma 2.1) that

$$
\frac{n-1}{4} \geq \sqrt{\frac{m}{8}+\frac{1}{64}}-\frac{1}{8}
$$

giving the required bound (2.2).

The bound of Edwards (2.2) is sharp for complete graphs of odd order. It came as a surprise when Alon [3], answering a question of Erdős [23], showed that (2.2) can be arbitrarily far from $f(m)$. More specifically, he proved that if $m=n_{0}^{2} / 2$ (half way between $\left(\begin{array}{c}n_{0} \\ 2\end{array}\right)$ and $\left.\left(\begin{array}{c}n_{0}+1 \\ 2\end{array}\right)\right)$ then

$$
f(m) \geq \frac{m}{2}+\sqrt{\frac{m}{8}}+c m^{1 / 4}
$$

on the other hand, unions of complete graphs show that, for every $m$,

$$
f(m) \leq \frac{m}{2}+\sqrt{\frac{m}{8}}+O\left(m^{1 / 4}\right) .
$$

A further improvement was obtained more recently. Alon and Halperin [4] and Bollobás and Scott [14] independently found a recursion for $f_{w}(m)$, showing that for sufficiently large $n$, and any $0 \leq k<n$, if $m=\left(\begin{array}{l}n \\ 2\end{array}\right)+k$ then

$$
f_{w}(m)=\min \left\{\left\lfloor\frac{(n+1)^{2}}{4}\right\rfloor,\left\lfloor\frac{n^{2}}{4}\right\rfloor+f_{w}(k)\right\} .
$$

The bound can be attained by taking either a copy of $K_{n+1}$ with some edges removed, or a copy of $K_{n}$ together with an extremal multigraph with $k$ edges (see [14] for further discussion of extremal graphs). Since it holds only for sufficiently large $n,(2.6)$ does not determine $f_{w}(m)$ for every $m$ (although Alon and Halperin [4] conjecture that (2.6) holds for every $m$ ).

It was noted in [14], as a consequence of (2.6), that there is a constant $C$ such that

$$
\left|f(m)-f_{w}(m)\right| \leq C \quad \text { for all } m \geq 1
$$

and so (2.6) determines $f(m)$ to within a constant for every $m$. In fact, the following conjecture was made both by Alon and Halperin [4] and in [14].

Conjecture $2.4 f(m)=f_{w}(m)$ for every $m$.

Note that it follows from (2.6) and (2.7) that the functions $f(m)$ and $f_{w}(m)$ are not very 'smooth': for instance, although $f(m) \sim f_{w}(m) \sim m / 2$, there are intervals of length $\Omega\left(m^{1 / 4}\right)$ on which both functions are constant.

Although we do not know the value of $f_{w}(m)$ for every $m$, there is an efficient algorithm that will always find a cut of at least this size.

Theorem 2.5 ([14]) There is an algorithm that, given a multigraph with $m$ edges, will find a cut of weight at least $f_{w}(m)$ in linear time. 
If Conjecture 2.4 is true, this would give an algorithm for finding a cut of size at least $f(m)$ in every graph $G$ with $m$ edges. Further discussion, and related results concerning fixed-parameter tractability and weak approximation algorithms, can be find in [14]. For a more general discussion of algorithms, see Laurent [31] and Poljak and Tuza [38].

Let us note briefly that for many classes of graph there are better bounds than $f(m)$. For instance, extending results of Poljak and Tuza [39] and Shearer [44] (see also Erdős [24], where the problem is raised, and Erdős, Faudree, Pach and Spencer [25]), Alon [3] proved that if $G$ is triangle-free then

$$
f(G) \geq \frac{m}{2}+c m^{4 / 5},
$$

while for every $m>0$ there is a triangle-free graph with $m$ edges such that

$$
f(G) \leq \frac{m}{2}+c^{\prime} m^{4 / 5}
$$

Thus the $m^{1 / 2}$ term in (2.2) is replaced in this case by an $m^{4 / 5}$ term. Alon, Bollobás, Krivelevich and Sudakov [1] proved that, for $r \geq 4$, if $G$ has girth at least $r$ then

$$
f(G) \geq \frac{m}{2}+c m^{r /(r+1)},
$$

and showed that, for every $m$, there is a graph with $m$ edges and girth at least 5 such that

$$
f(G) \leq \frac{m}{2}+c^{\prime} m^{5 / 6}
$$

They conjecture that a similar result should hold for every $r \geq 4$. Further results of this type can be found in Alon, Krivelevich and Sudakov [5], and a detailed discussion can be found in Poljak and Tuza [38].

\subsection{Max $k$-cut}

Let us write $f_{k}(G)$ for the maximum number of edges in a $k$-partite subgraph of $G$, and define

$$
f_{k}(m)=\min \left\{f_{k}(G): e(G)=m\right\} .
$$

We define $\tilde{f}_{k}$ to be the same quantity for multigraphs (so $\tilde{f}_{2}(m) \equiv f_{w}(m)$ ). Clearly

$$
f_{k}(m) \geq \tilde{f}_{k}(m) .
$$

By considering a random partition into $k$ classes, it is easy to see that

$$
f_{k}(m) \geq \frac{k-1}{k} m,
$$

while an analogue to the Edwards bound was proved in [14]:

$$
\tilde{f}_{k}(m) \geq\left(1-\frac{1}{k}\right) m+\frac{k-1}{2 k} \sqrt{2 m+1 / 4}+\frac{k^{2}-2 k+2}{8},
$$

with equality when $G$ is a complete graph.

It was shown in [14] that if $\delta(G)=\omega(n)$, where $\omega(n) \rightarrow \infty$ as $n \rightarrow \infty$, then

$$
f_{k}(G) \geq \frac{k-1}{k} m+\frac{k-1}{2 k} n+o(n),
$$

which is sharp except for the $o(n)$ term. A sharper result was conjectured. 
Conjecture 2.6 ([14]) If $G$ is $(k-1)$-connected then

$$
f_{k}(G) \geq \frac{k-1}{k} m+\frac{k-1}{2 k} n+O(1) .
$$

It is also possible (see [14]) to extend (2.6) to the $k$-partite context: the functions $\tilde{f}_{k}(m)$ and $f_{k}(m)$ turn out to have similar properties to $f_{w}(m)$ and $f(m)$.

\subsection{Max Bisection}

The extremal theory for Max Cut is now quite well developed. For Max Bisection, however, much less is known. Bisection problems are more restricted than cut problems, and the available tools are consequently more limited. For instance, the 'repeated contraction' argument used for Theorem 2.1 does not work, as we need to keep track of the number of vertices on each side of the partition.

Let us write $b(G)$ for the maximum of $e\left(V_{1}, V_{2}\right)$ over all partitions $V(G)=V_{1} \cup V_{2}$ with $\left|V_{1}\right| \leq\left|V_{2}\right| \leq\left|V_{1}\right|+1$, and define

$$
b(m)=\min \{b(G): e(G)=m\} .
$$

By considering random bipartitions, it is clear that

$$
b(m) \geq\lceil m / 2\rceil .
$$

On the other hand, this bound is clearly achieved by the star $K_{1, n}$. However, it is less clear what happens if the graph is not so sparse.

Problem 2.7 Fix $\delta>1$. What is

$$
\min \{b(G): e(G)=m, \delta(G) \geq \delta\} ?
$$

Natural families of graphs to consider here are the complete bipartite graph $K_{\delta, n}$ and the graphs $K_{\delta}+E_{n}$ obtained from $K_{\delta, n}$ by filling in one vertex class.

A similar problem arises if we restrict the number of vertices.

Problem 2.8 For $\delta \geq 1$. For $m, n \geq 1$, what is

$$
\min \{b(G): e(G)=m,|G|=n\} ?
$$

An interesting range here is when $n=O(\sqrt{m})$. Considering a random bisection shows that in this range we get $b(G) \geq m / 2+\Omega(\sqrt{m})$. But how close do we get to the Edwards bound (2.2)?

\section{Judicious partitions of graphs}

\subsection{Each class contains few edges}

We now turn to considering various types of judicious partition. For a graph $G$, we define

$$
g(G)=\min _{V(G)=V_{1} \cup V_{2}} \max \left\{e\left(V_{1}\right), e\left(V_{2}\right)\right\} .
$$


For $m \geq 1$, we define

$$
g(m)=\max _{e(G)=m} g(G) .
$$

Thus $g(m)$ is the smallest integer such that every graph $G$ with $m$ edges has a bipartition in which each class contains at most $g(m)$ edges.

It is easily seen that

$$
g(m) \leq m / 3 .
$$

Indeed, consider a maximum cut $V(G)=V_{1} \cup V_{2}$. Clearly every $v \in V_{1}$ has

$$
\left|\Gamma(v) \cap V_{2}\right| \geq\left|\Gamma(v) \cap V_{1}\right|,
$$

or else we could increase the size of the cut by moving $v$ from $V_{1}$ to $V_{2}$. Summing (3.2) over vertices in $V_{1}$, we get

$$
\begin{aligned}
2 e\left(V_{1}\right) & =\sum_{v \in V_{1}}\left|\Gamma(v) \cap V_{1}\right| \\
& \leq \sum_{v \in V_{1}}\left|\Gamma(v) \cap V_{2}\right| \\
& =e\left(V_{1}, V_{2}\right),
\end{aligned}
$$

and so $e\left(V_{1}\right) \leq m / 3$. Similarly, for $v \in V_{2}$, we have

$$
\left|\Gamma(v) \cap V_{1}\right| \geq\left|\Gamma(v) \cap V_{2}\right|,
$$

and the same argument shows that $e\left(V_{2}\right) \leq m / 3$.

Bipartitions such that every $v \in V_{1}$ satisfies (3.2) and every $v \in V_{2}$ satisfies (3.3) are called unfriendly. As we have noted, it is very easy to see that every finite graph has an unfriendly bipartition, but for infinite graphs the picture is quite different. Aharoni, Milner and Prikry [2] showed that unfriendly bipartitions exist for certain classes of infinite graphs. Shelah and Milner [45] proved the following surprising result.

Theorem 3.1 ([45]) There is a graph with $\left(2^{\omega}\right)^{(+\omega)}$ vertices and no unfriendly bipartition.

On the other hand, there is a positive result for partitions into more than two classes. We say that a vertex-partition of a graph $G$ (into any number of classes) is unfriendly if every vertex has at least as many neighbours in every other class as in its own class.

Theorem 3.2 ([45]) For $k \geq 3$, every graph has an unfriendly partition into $k$ sets.

Returning to finite graphs, the bound $g(m) \leq m / 3$ is attained with equality only by $K_{3}$ (and this is the only extremal graph). But what happens for larger $m$ ? For Max Cut, it is easy to show that $f(m) \sim m / 2$, but the asymptotics of $g(m)$ are less easy to determine.

A number of papers gave successively better bounds for $g(m)$. It was shown in [18] that

$$
g(m) \leq \frac{m}{4}+c m^{4 / 5} .
$$


Porter [42] determined the correct order of magnitude for the second term, showing that

$$
g(m) \leq \frac{m}{4}+\sqrt{2} m
$$

and Porter and Bin Yang [43] showed that

$$
g(m) \leq \frac{m}{4}+\sqrt{\frac{8 m}{9}} .
$$

Finally, a sharp result was proved in [16], which gave the following analogue of (2.2). Surprisingly, it turned out that this could be combined with a partition giving a large cut.

Theorem 3.3 ([16]) Every graph $G$ with $m$ edges has a bipartition $V(G)=V_{1} \cup V_{2}$ such that

$$
e\left(V_{1}, V_{2}\right) \geq \frac{m}{2}+\sqrt{\frac{m}{8}+\frac{1}{64}}-\frac{1}{8}
$$

and, for $j=1,2$,

$$
e\left(V_{i}\right) \leq \frac{m}{4}+\sqrt{\frac{m}{32}+\frac{1}{256}}-\frac{1}{16} .
$$

The extremal graphs are the complete graphs of odd order.

Note that (3.5) is exactly half of the Edwards bound (3.4).

Proof We give a sketch of the proof. Let us start with a maximum cut $V(G)=$ $V_{1} \cup V_{2}$, and suppose that $e\left(V_{1}\right) \geq e\left(V_{2}\right)$. If $V_{1}$ satisfies (3.5), we are done. Otherwise, we successively move vertices from $V_{1}$ to $V_{2}$ until we obtain $V_{1}^{\prime} \subset V_{1}$ that satisfies (3.5).

Suppose we have reached a partition $\left(W_{1}, W_{2}\right)$, where $W_{1} \subset V_{1}$ but $W_{1}$ does not satisfy (3.5). We pick $w \in W_{1}$ with $\left|\Gamma(w) \cap W_{1}\right|$ as small as possible, and move it across. Let $\left(W_{1}^{\prime}, W_{2}^{\prime}\right)$ be the resulting partition. We claim that $W_{2}^{\prime}$ satisfies (3.5) and $\left(W_{1}^{\prime}, W_{2}^{\prime}\right)$ satisfies (3.4). The theorem will then be proved, as we continue moving vertices until $W_{1}^{\prime}$ first satisfies (3.5).

First of all, note that for $v \in V_{1}$ we have $\left|\Gamma(v) \cap V_{2}\right| \geq\left|\Gamma(v) \cap V_{1}\right|$, since $\left(V_{1}, V_{2}\right)$ is a maximum cut. Since we are only moving vertices from $V_{1}$ to $V_{2}$, we have for all $v \in W_{1}$,

$$
\left|\Gamma(v) \cap W_{1}\right| \leq\left|\Gamma(v) \cap W_{2}\right| .
$$

Now suppose that

$$
e\left(W_{1}\right)=\frac{m}{4}+\alpha,
$$

where

$$
\alpha>\sqrt{\frac{m}{32}+\frac{1}{256}}-\frac{1}{16} .
$$


Recall that $w$ is the vertex we move from $W_{1}$ to $W_{2}$ and let $\delta=\left|\Gamma(w) \cap W_{1}\right|$. Then

$$
\begin{aligned}
e\left(W_{1}^{\prime}, W_{2}^{\prime}\right) & =\sum_{v \in W_{1}^{\prime}}\left|\Gamma(v) \cap W_{2}^{\prime}\right| \\
& =\sum_{v \in W_{1}^{\prime}}\left|\Gamma(v) \cap W_{2}\right|+\delta \\
& \geq \sum_{v \in W_{1} \backslash w}\left|\Gamma(v) \cap W_{1}\right|+\delta \\
& =\left(2 e\left(W_{1}\right)-\delta\right)+\delta \\
& =\frac{m}{2}+2 \alpha,
\end{aligned}
$$

which satisfies (3.4), as $\alpha>\sqrt{m / 32+1 / 256}-1 / 16$.

Since $\delta=\delta\left(G\left[W_{1}\right]\right)=\left|\Gamma(w) \cap W_{1}\right|$, we have

$$
e\left(W_{1}\right) \geq\left(\begin{array}{c}
\delta+1 \\
2
\end{array}\right)
$$

and so

$$
\delta \leq \sqrt{2 e\left(W_{1}\right)+\frac{1}{4}}-\frac{1}{2}=\sqrt{\frac{m}{2}+2 \alpha+\frac{1}{4}}-\frac{1}{2} .
$$

So

$$
\begin{aligned}
e\left(W_{2}^{\prime}\right) & =m-e\left(W_{1}^{\prime}\right)-e\left(W_{1}^{\prime}, W_{2}^{\prime}\right) \\
& \leq m-\left(\frac{m}{4}+\alpha-\delta\right)-\left(\frac{m}{2}+2 \alpha\right) \\
& =\frac{m}{4}-3 \alpha+\delta \\
& \leq \frac{m}{4}-3 \alpha+\sqrt{\frac{m}{2}+2 \alpha+\frac{1}{4}}-\frac{1}{2},
\end{aligned}
$$

which is bounded above by (3.5) for $\alpha>\sqrt{m / 32+1 / 256}-1 / 16$.

A more general version of the argument produces a 'biased' extension of Theorem 3.3 .

Theorem 3.4 ([16]) Let $G$ be a graph with $m$ edges and let $0 \leq p \leq 1$. There is a partition $V(G)=V_{1} \cup V_{2}$ such that

$$
e\left(V_{1}\right) \leq p^{2} m+c(p, m)
$$

and

$$
e\left(V_{2}\right) \leq(1-p)^{2} m+c(p, m)
$$

where

$$
c(p, m)=p(1-p)(\sqrt{m / 2+1 / 16}-1 / 4) .
$$


We now turn to the $k$-partite version of the question. Let

$$
g_{k}(G)=\min _{V(G)=V_{1} \cup \cdots \cup V_{k}} \max \left\{e\left(V_{1}\right), \ldots, e\left(V_{k}\right)\right\} .
$$

For $m \geq 1$, we define

$$
g_{k}(m)=\max _{e(G)=m} g_{k}(G) .
$$

It was shown in [18] that

$$
g_{k}(m) \leq m /\left(\begin{array}{c}
k+1 \\
2
\end{array}\right)
$$

which is best possible for the complete graph $K_{k+1}$. However, as in the bipartite case, it is possible to do much better for larger graphs. It was proved in [18] that

$$
g(m) \leq \frac{m}{k^{2}}+O\left(m^{4 / 5}\right),
$$

while Porter [41] showed that if $k$ is a power of 2 then

$$
g_{k}(m) \leq \frac{m}{k^{2}}+\sqrt{\frac{m}{k}} .
$$

Porter [40] showed that, for every $k$,

$$
g_{k}(m) \leq \frac{m}{k^{2}}+4 k \sqrt{m},
$$

and Porter and Bin Yang [43] showed that, for $k$ a power of 2,

$$
g_{k}(m) \leq \frac{m}{k^{2}}+\frac{1.31 \sqrt{m}}{k} .
$$

As in the bipartite case, a sharp result was proved in [16] (using Theorem 3.4 as an essential tool).

Theorem 3.5 ([16]) Every graph with $m$ edges has a vertex-partition into $k$ sets, each of which contains at most

$$
\frac{m}{k^{2}}+\frac{k-1}{2 k^{2}}\left(\sqrt{2 m+\frac{1}{4}}-\frac{1}{2}\right)
$$

edges.

The extremal graphs are complete graphs of order $k n+1$. However, unlike Theorem 3.3, it is not known whether a $k$-partition satisfying (3.6) can be achieved with a large cut.

Problem 3.6 ([13]) Does every graph with $m$ edges have a partition into $k$ sets that satisfies both (3.6) and (2.8)? 
A starting point for this would be to prove that there is a $k$-cut that satisfies (3.6) and has size at least $(1-1 / k) m$. (Although note that when $k$ is a power of 2 , we can apply Theorem 3.3 recursively.)

The bounds given by (3.5) and (3.4) are both optimal for complete graphs of odd order. It would be interesting to know the behaviour of

$$
\frac{m}{4}+\sqrt{\frac{m}{32}}-g(m)
$$

For instance, it seems very likely that $m / 4+\sqrt{m / 32}-g(m)$ is unbounded, as conjectured in [13]. Perhaps the following judicious version of Alon's result (2.5) is true.

Conjecture 3.7 There is some $c>0$ such that

$$
g(m)<\frac{m}{4}+\sqrt{\frac{m}{32}}-c m^{1 / 4}
$$

for infinitely many values of $m$.

As with Max Cut, a good starting point might be to consider graphs with $m$ about half way between $\left(\begin{array}{c}n \\ 2\end{array}\right)$ and $\left(\begin{array}{c}n+1 \\ 2\end{array}\right)$, or else of form $\left(\begin{array}{c}2 t \\ 2\end{array}\right)$.

More generally, it would be desirable to pin down $g(m)$ more precisely.

Problem 3.8 Is there a recursion for $g(m)$, for $m$ sufficiently large, analogous to $(2.6)$ ?

The same problem arises in the $k$-partite case: if $m$ is not of form $\left(\begin{array}{c}2 n+1 \\ 2\end{array}\right)$, how far out can (3.6) be? For instance, Hofmeister and Lefmann [28] proved that if $G$ has $\left(\begin{array}{c}k n \\ 2\end{array}\right)$ edges then it has a partition into $k$ vertex classes $V_{1}, \ldots, V_{k}$ with $\sum_{i=1}^{k} e\left(V_{i}\right) \leq$ $k\left(\begin{array}{l}n \\ 2\end{array}\right)=n(k n-k) / 2$, beating the trivial bound of $(1 / k)\left(\begin{array}{c}n k \\ 2\end{array}\right)=n(k n-1) / 2$ by $(k+1) n / 2$.

Problem 3.9 ([16]) Does every graph $G$ with $\left(\begin{array}{c}k n \\ 2\end{array}\right)$ edges have a vertex partition into $k$ sets, each of which contains at most $\left(\begin{array}{l}n \\ 2\end{array}\right)$ edges?

Finally, we note that the proof of Theorem 3.3 gives a polynomial-time algorithm that finds a partition satisfying (3.4) and (3.5) (we start with a partition satisfying (3.2) and (3.3), rather than with a maximum cut). It would be nice to have a judicious version of Theorem 2.5 .

Problem 3.10 Is there a polynomial-time algorithm that takes as input a graph with $m$ edges and finds a partition $V(G)=V_{1} \cup V_{2}$ with $\max \left\{e\left(V_{1}\right), e\left(V_{2}\right)\right\} \leq g(m)$ ?

\subsection{Bounded-degree graphs}

For graphs with bounded maximal degree, we can get much stronger results. Indeed, the constant in the linear term in (3.5) can be improved.

The following was shown in [12]. 
Theorem 3.11 ([12]) If $k$ is odd, then every graph $G$ with $m$ edges and $\Delta(G) \leq k$ has a bipartition $V(G)=V_{1} \cup V_{2}$ with

$$
\max \left\{e\left(V_{1}\right), e\left(V_{2}\right)\right\} \leq \frac{k-1}{4 k} m+\frac{k-1}{4}
$$

and

$$
e\left(V_{1}, V_{2}\right) \geq \frac{k+1}{2 k} m .
$$

The extremal graphs for (3.7) are of form $(2 t+1) K_{k} \cup s K_{k+1}$. Note that if $k$ is even, then an optimal bound is obtained by applying the theorem with $k+1$ as the degree bound: the extremal graphs are of form $(2 t+1) K_{k+1}$.

For graphs that are regular, we can do even better. If the maximal degree is odd we get the following.

Theorem 3.12 ([12]) Let $k \geq 1$ be odd, and suppose $G$ is a $k$-regular graph with $m$ edges. Then there is a partition $V(G)=V_{1} \cup V_{2}$ with $\left|V_{1}\right|=\left|V_{2}\right|$ and

$$
\max \left\{e\left(V_{1}\right), e\left(V_{2}\right)\right\} \leq \frac{k-1}{4 k} m .
$$

The extremal graphs are of form $s K_{k+1}$.

If the maximal degree is even, we cannot do much better.

Theorem 3.13 ([12]) Let $k \geq 2$ be even, and suppose $G$ is a k-regular graph with $m$ edges.

(a) If $|G|$ is even, there is a partition $V(G)=V_{1} \cup V_{2}$ with $\left|V_{1}\right|=\left|V_{2}\right|$ and

$$
\max \left\{e\left(V_{1}\right), e\left(V_{2}\right)\right\} \leq \frac{k}{4(k+1)} m .
$$

The extremal graphs are of form $2 t K_{k+1}$.

(b) If $|G|$ is odd, there is a partition $V(G)=V_{1} \cup V_{2}$ with $\left|V_{2}\right|=\left|V_{1}\right|+1$ and

$$
\max \left\{e\left(V_{1}\right), e\left(V_{2}\right)\right\} \leq \frac{1}{4} \frac{k}{k+1} m+\frac{k}{4}
$$

The extremal graphs are of form $(2 t+1) K_{k+1}$.

The extremal graphs for Theorem 3.11, 3.12 and 3.13 are all unions of complete graphs. We should expect to be able to do rather better for graphs without large cliques.

Problem 3.14 ([12]) For $k \geq 3$, what are the optimal constants $c_{k}$ and $d_{k}$ such that every $k$-regular $K_{k+1}$-free graph with $m$ edges has $f(G) \geq c_{k} m$ and $g(G) \geq d_{k} m$ ?

Note that if $G$ is $k$-regular then any partition $V(G)=V_{1} \cup V_{2}$ with $\left|V_{1}\right|=\left|V_{2}\right|$ has $e\left(V_{1}\right)=e\left(V_{2}\right)$, so Max Bisection gives a bound on $g(G)$. For instance, for $k=3$, Locke [34] showed that every cubic $K_{4}$-free graph has a bisection of size at least 
$11 e(G) / 15$; it follows that $g(G) \leq 2 e(G) / 15$, which is better than the bound $e(G) / 6$ from Theorem 3.12.

For graphs with larger girth, it should be possible to get stronger results (see $[13,19,29,34,46])$. One approach here is to combine a good colouring with Theorem 2.2. For graphs without large cliques, there are powerful strengthenings of Brooks' Theorem that provide colourings with fewer than $\Delta(G)$ colours (see Molloy and Reed [35] for a detailed discussion). For triangle-free graphs, Johansson [30] showed that $\chi(G)=O(\Delta / \log \Delta)$; it follows that $f(G) \geq m / 2+O(m \log \Delta / \Delta)$. However, the resulting colouring might be very imbalanced, and so this does not help us with $g(G)$. (Note that the Hajnal-Szemerédi Theorem [27] on balanced colourings does not quite give Theorem 3.12.) Further discussion can be found in [13].

Finally, we note that, for graphs with bounded degrees, there are partitions that satisfy very strong conditions.

Theorem 3.15 ([12]) Let $k \geq 2$. For every $\Delta \geq 1$ there is a constant $K=K(\Delta)$ such that for every graph $G$ with $\Delta(G) \leq \Delta$ and every sequence $p_{1}, \ldots, p_{k}$ of nonnegative reals with $\sum_{i=1}^{k} p_{i}=1$, there is a partition $V(G)=\bigcup_{i=1}^{k} V_{i}$ such that, for every $i$,

$$
|| V_{i}\left|-p_{i}\right| G|| \leq K
$$

and

$$
\left|e\left(V_{i}\right)-p_{i}^{2} e(G)\right| \leq K
$$

and, for every $i \neq j$,

$$
\left|e\left(V_{i}, V_{j}\right)-p_{i} p_{j} e(G)\right| \leq K .
$$

A similar result is proved in [12] for hypergraphs.

\subsection{Judicious partitions and maximum cuts}

Given a graph $G$, a trivial bound on $g(G)$ is given by the fact that

$$
e\left(V_{1}, V_{2}\right) \geq m-2 \max \left\{e\left(V_{1}\right), e\left(V_{2}\right)\right\} .
$$

It follows immediately that

$$
f(m)+2 g(m) \geq m
$$

and, by Theorem 3.3, we know the value of $f(m)+2 g(m)$ whenever $m$ has form $\left(\begin{array}{l}n \\ 2\end{array}\right)$. However, it is unclear what happens for other values of $m$. Related to Conjecture 3.7 , we have the following problem.

Problem 3.16 What is the behavour of the function $f(m)+2 g(m)-m$ ?

This is expressed rather imprecisely, but the aim is to get a very precise description of the behaviour of $f(m)+2 g(m)$.

If we have a graph for which $g(G)$ is particularly small, then (3.9) implies that $f(G)$ is large. So graphs with small judicious partitions have large cuts. It is natural to wonder whether the converse is also true, and Alon, Bollobás, Krivelevich and Sudakov proved the following. 
Theorem 3.17 ([1]) Let $G$ be a graph with $m$ edges and suppose that $f(G)=\frac{m}{2}+\delta$. If $\delta \leq m / 30$ then

$$
g(G) \leq \frac{m}{4}-\frac{\delta}{2}+\frac{10 \delta^{2}}{m}+3 \sqrt{m}
$$

and if $\delta \geq m / 30$ then

$$
g(G) \leq \frac{m}{4}-\frac{m}{100}
$$

Note that if $f(G)=m / 2+\delta$ then $g(G) \geq m / 4-\delta / 2$, so the theorem is sharp up to the error term $10 \delta^{2} / m+3 \sqrt{m}$, which is $o(\delta)$ provided $\delta=o(m)$. It would be very interesting to have more exact bounds, although this might be quite difficult.

Note that Theorem 3.17 does not help when $\delta$ is $O(\sqrt{m})$, as the $3 \sqrt{m}$ error term overwhelms the gain of $\delta / 2$; it would be good to have sharper bounds in this range.

An extension of Theorem 3.17 for partitions into more than two parts was proved in $[10]$.

\subsection{Other norms}

Elsewhere we have considered Max Cut, which can be thought of as minimizing the $l_{1}$ norm of $\left(e\left(V_{1}\right), e\left(V_{2}\right)\right)$ over partitions $V(G)=V_{1} \cup V_{2}$; we have also considered the judicious partitioning problem of minimizing the $l_{\infty}$ norm of $\left(e\left(V_{1}\right), e\left(V_{2}\right)\right)$. However, we could equally well look for the minimum of other norms. For instance, we have the following natural problem.

Problem 3.18 What is the maximum of

$$
\min _{V(G)=V_{1} \cup V_{2}} e\left(V_{1}\right)^{2}+e\left(V_{2}\right)^{2}
$$

over graphs $G$ with $m$ edges?

Similar problems arise when we partition into more than two sets, or consider other norms.

In general, given an invariant $\mu$ of graphs, we can ask for the minimum of

$$
\mu\left(G\left[V_{1}\right]\right)+\mu\left(G\left[V_{2}\right]\right)
$$

or

$$
\max \left\{\mu\left(G\left[V_{1}\right]\right), \mu\left(G\left[V_{2}\right]\right)\right\}
$$

over partitions $V(G)=V_{1} \cup V_{2}$. Writing $f_{\mu}(G)$ and $g_{\mu}(G)$ for these two functions, we define corresponding functions

$$
f_{\mu}(m)=\min \left\{f_{\mu}(G): e(G)=m\right\}
$$

and

$$
g_{\mu}(m)=\min \left\{g_{\mu}(G): e(G)=m\right\} .
$$

For instance, if $\mu(G)=e(G)$ then determining $f_{\mu}(G)$ and $g_{\mu}(G)$ corresponds to Max Cut and the judicious partitioning problem considered in section 3 .

There are many problems, for instance the following. 
Problem 3.19 Let

$$
\mu(H)=\sum_{v \in V(H)} d_{H}(v)^{2} .
$$

What is $f_{\mu}(m)$ ? What is $g_{\mu}(m)$ ?

Of course, many other combinatorial problems can be expressed in this manner.

\subsection{Multiple graphs}

So far, we have been concerned with problems that involve partitions optimizing several quantities simultaneously for a given graph. We can also consider partitions that optimize a single quantity simultaneously for several different graphs defined on the same vertex set. For instance, the following question is raised in [12].

Problem 3.20 ([12]) Find the largest integer $f(m ; 2)$ such that for every pair of graphs $G_{1}, G_{2}$, with $e\left(G_{1}\right)=e\left(G_{2}\right)=m$ and $V\left(G_{1}\right)=V\left(G_{2}\right)$, there is a partition $V\left(G_{i}\right)=V_{1} \cup V_{2}$ such that

$$
\min \left\{e_{G_{1}}\left(V_{1}, V_{2}\right), e_{G_{2}}\left(V_{1}, V_{2}\right)\right\} \geq f(m ; 2) .
$$

A starting point for this question is to determine whether

$$
f(m ; 2)=(1+o(1)) m / 2 .
$$

Of course, there are many interesting variations on the question. For instance, what if there are more than two graphs? What about partitions such that $\max \left\{e_{G_{1}}\left(V_{1}\right)\right.$, $\left.e_{G_{1}}\left(V_{2}\right)\right\}$ and $\max \left\{e_{G_{2}}\left(V_{1}\right), e_{G_{2}}\left(V_{2}\right)\right\}$ are both small?

There is also an interesting relationship between the maximum cut in a graph $G$ and the maximum cut of its complement. For a graph $G$ with $m$ edges, we define

$$
f^{+}(G)=f(G)-m / 2 .
$$

The following was proved in [9] (see also [11]).

Theorem 3.21 ([9]) If $G$ is a graph with $n$ vertices and $p\left(\begin{array}{l}n \\ 2\end{array}\right)$ edges, where $4 / n \leq$ $p \leq 1-4 / n$ then

$$
f^{+}(G) f^{+}(\bar{G}) \geq c(p) n^{3} .
$$

We can think of Theorem 3.21 as a result about 2-colourings of the edges of $K_{n}$. What can we say if we use more than two colours?

\subsection{Judicious bisections}

As with the extremal version of Max Bisection, comparatively little is known about judicious partitions in which we demand that the partition is balanced, i.e. the vertex classes are as equal as possible in size. Considering $K_{1, n-1}$ shows that we cannot in general demand a balanced bipartition with fewer than $\lfloor m / 2\rfloor$ edges in each vertex class. However, it may be possible to do better for graphs that are denser. 
Problem 3.22 ([13]) What is the smallest $c(k)$ such that every graph $G$ with $m$ edges and minimal degree $k$ has a bisection with at most $c(k) m$ edges in each vertex class?

It is easily seen that $c(1)=1 / 2$ : given $G$, consider a bipartition $V(G)=$ $V_{1} \cup V_{2}$ chosen uniformly at random from all bipartitions with $\left|V_{1}\right|=\left|V_{2}\right|$ (if $|G|$ is odd then add an isolated vertex). Then $\mathbb{E} e\left(V_{1}\right)<m / 4$, and so $\mathbb{P}\left(e\left(V_{1}\right) \geq\right.$ $m / 2)<1 / 2$, and similarly for $e\left(V_{2}\right)$. So with positive probability the bipartition has $\max \left\{e\left(V_{1}\right), e\left(V_{2}\right)\right\}<m / 2$. We deduce that $c(1) \leq 1 / 2$. On the other hand, as noted above, $K_{1, n-1}$ shows that $c(1) \geq 1 / 2$.

Considering graphs of form $K_{3, n-3}$ shows that $c(2) \geq c(3) \geq 1 / 3$.

Conjecture $3.23([13]) c(2)=1 / 3$.

A starting point for the problem might be the following conjecture from [13], which asserts that every graph has a bisection that is close to an unfriendly partition.

Conjecture 3.24 ([13]) Every graph $G$ has a bisection $V(G)=V_{1} \cup V_{2}$ such that

$$
\begin{array}{ll}
\left|\Gamma(v) \cap V_{1}\right| \leq\left|\Gamma(v) \cap V_{2}\right|+1 & \forall v \in V_{1} \\
\left|\Gamma(v) \cap V_{2}\right| \leq\left|\Gamma(v) \cap V_{1}\right|+1 & \forall v \in V_{2} .
\end{array}
$$

If true, this could not be improved, as can be seen by considering graphs of form $K_{2 k+1,2 l+1}$, where $k \neq l$.

A different condition that might ensure a good judicious bipartition is $\Delta(G)=$ $o(n)$, as suggested in [13]. We make the following conjecture.

Conjecture 3.25 If $G$ is a graph with $n$ vertices and $\Delta(G)=o(n)$ then there is a bisection $V(G)=V_{1} \cup V_{2}$ such that

$$
\max \left\{e\left(V_{1}\right), e\left(V_{2}\right)\right\} \leq(1+o(1)) e(G) / 4 .
$$

\subsection{Digraphs}

There is also a version of the Max Cut problem for digraphs. For a directed graph $D$, we define

$$
d f(D)=\max _{V(D)=V_{1} \cup V_{2}} e\left(V_{1}, V_{2}\right)
$$

and

$$
d f(m)=\min \{d f(D): e(D)=m\} .
$$

It is easily seen that $d f(D) \geq f(G) / 2$, where $G$ is the underlying multigraph of $D$ (if $x y$ and $y x$ are edges then $x y$ will be a double edge in $G$ ). Thus

$$
d f(m) \geq f_{w}(m) / 2 .
$$

The behaviour of $d f(m)$ is closely related to the behaviour of $f(m)$, as shown in [14].

It is natural to ask what happens if we restrict our attention to acyclic directed graphs. Surprisingly, we still get a cut of almost the same size. 
Theorem 3.26 ([7]) For every $m \geq 1$, there is an acyclic digraph $D$ with $m$ edges and

$$
d f(D) \geq(1+o(1)) m / 4 .
$$

A large directed cut asks only for $e\left(V_{1}, V_{2}\right)$ to be large. An interesting judicious partitioning problem arises when we ask for both $e\left(V_{1}, V_{2}\right)$ and $e\left(V_{2}, V_{1}\right)$ to be large. The star $K_{1, n-1}$ with all edges directed away from the centre shows that we need some degree condition.

Problem 3.27 Fix $k>0$. What is the largest constant $c(k)$ such that every digraph with $m$ edges and minimum outdegree at least $k$ has a bipartition $V(D)=V_{1} \cup V_{2}$ with

$$
\min \left\{e\left(V_{1}, V_{2}\right), e\left(V_{2}, V_{1}\right)\right\} \geq c(k) m ?
$$

The same problem arises for partitions into more than two parts.

\section{Hypergraphs}

\subsection{Each class contains few edges}

As with most combinatorial problems, judicious partitioning problems are typically much harder for hypergraphs than for graphs, and correspondingly much less is known. We give just a brief account, concentrating on two types of problem.

We extend our notation as follows. For a hypergraph $H$, and $X \subset V(H)$, we write $e(X)$ for the number of edges contained in $X$. For disjoint subsets $X, Y$ of $V(H)$, we write $e(X, Y)$ for the number of edges contained in $X \cup Y$ and incident with both $X$ and $Y$. So $e(X, Y)=e(X \cup Y)-e(X)-e(Y)$. Then, for a hypergraph $H$, we define

$$
f(H)=\max _{V(H)=V_{1} \cup V_{2}} e\left(V_{1}, V_{2}\right),
$$

where the maximum is taken over partitions $V(H)=V_{1} \cup V_{2}$, and

$$
f^{(k)}(m)=\min \{f(H): H \text { a } k \text {-uniform hypergraph, } e(H)=m\} .
$$

Similarly,

$$
g(H)=\min _{V(H)=V_{1} \cup V_{2}} \max \left\{e\left(V_{1}\right), e\left(V_{2}\right)\right\}
$$

and

$$
g^{(k)}(m)=\max \{g(H): H \text { a } k \text {-uniform hypergraph, } e(H)=m\} .
$$

The extremal theory is much less developed than for graphs. For instance, it is easy to show that

$$
f^{(k)}(m)=\left(1-2^{1-k}\right) m+o(m),
$$

but the following is open even for $k=3$.

Problem 4.1 Prove a bound for k-uniform hypergraphs analogous to (2.2).

A natural conjecture is that, for $m$ of form $\left(\begin{array}{l}n \\ k\end{array}\right)$, the complete $k$-uniform hypergraph should be extremal.

For $g^{(k)}(m)$, even less is known, The following result was proved in [17]. 
Theorem 4.2 ([17]) Every 3-uniform hypergraph with $m$ edges has a partition into $k$ sets, each of which contains at most

$$
(1+o(1)) m / k^{3}
$$

edges.

The error term given in [17] was of size $O\left(\mathrm{~m}^{6 / 7}\right)$, although it seems likely that the truth should be $O\left(\mathrm{~m}^{2 / 3}\right)$. The difficulty comes from the fact that we are optimizing several variables simultaneously: in a random $k$-partition we expect $m / k^{3}$ edges in each vertex class. If there are no large-degree vertices, then we can prove this immediately by taking a random partition and using a martingale inequality. The obstacle is that there may be vertices of large degree, and this is dealt with by first partitioning the large-degree vertices using an extremal argument and then handling the rest of the graph with probabilistic techniques.

It seems likely that a similar result should hold for $r$-uniform hypergraphs, and it seems surprising that it still remains open.

Conjecture 4.3 ([17]) Let $r \geq 3$ and $k \geq 2$ be fixed integers. Then every $r$-uniform hypergraph with $m$ edges has a vertex-partition into $k$ classes, each of which contains at most

$$
\frac{m}{k^{r}}+o(m)
$$

edges.

Perhaps an even stronger conjecture may be true.

Conjecture 4.4 ([17]) Let $r \geq 3$. Every $r$-uniform hypergraph with $m$ edges has a bipartition with at most $m / 2^{r}$ edges in each class.

In other words, $g^{(r)}(m) \leq m / 2^{r}$ for $r \geq 3$. This would be very surprising if true, since it would imply the existence of a bipartition in which both sides simultaneously beat the expected number of edges in a random bipartition. Note that the conjecture would not hold for $r=2$, since we have $g(m)=m / 2+\Omega(\sqrt{m})$. Even if the conjecture is not true, there may only be finitely many counterexamples.

The best current bound is the following.

Theorem 4.5 ([13]) Let $r \geq 2$ and $k \geq 2$. Every $r$-uniform hypergraph with $m$ edges has a vertex partition into $k$ classes with at most

$$
a_{r} \frac{m}{k^{r}}+b_{r} m^{2 r /(2 r+1)}
$$

edges in each class, where $a_{r}=O(r / \log r)$.

Finally, it seems likely that the relationship between $f^{(k)}(G)$ and $g^{(k)}(G)$ should hold, as in the graph context. We raise the following problem.

Problem 4.6 Prove a version of Theorem 3.17 for 3-uniform hypergraphs. 


\subsection{Each class meets many edges}

What about hypergraph partitions in which each class meets many edges? Bollobás and Thomason (see $[8,15]$ ) conjectured the following.

Conjecture 4.7 For $r \geq 3$, every $r$-uniform hypergraph with $m$ edges has a partition into $r$ vertex classes, each of which meets at least

$$
\frac{r m}{2 r-1}
$$

edges.

For $r=2$, the statement is the same as (3.1), and so is easily proved. For $r \geq 3$, Bollobás, Reed and Thomason [8] showed that there is a partition in which every class meets at least

$$
\left(1-\frac{1}{e}\right) m / 3 \approx 0.21 m
$$

edges. It was shown in [15] that every 3-uniform hypergraph has a tripartition in which each class meets at least

$$
(5 m-1) / 9
$$

edges, which is still short of the conjectured $3 \mathrm{~m} / 5$; for $r$-uniform hypergraphs, a lower bound of

$$
0.27 m
$$

was given. It would be a substantial step forward even to prove a lower bound of form $m / 2$.

Although Conjecture 4.7 is easily proved when $r=2$, it seems much harder to find a $k$-partite version of the result. The following is a version of a conjecture from [15].

Conjecture 4.8 For every $k \geq 2$, every graph with $m \geq\left(\begin{array}{l}k \\ 2\end{array}\right)$ edges has a partition into $k$ sets, each of which meets at least

$$
\frac{2 m}{2 k-1}
$$

edges.

The lower bound on $m$ is necessary to avoid trivial cases such as $K_{k-1}$, where one vertex class may end up empty.

\section{References}

[1] N. Alon, B. Bollobás, M. Krivelevich and B. Sudakov, Maximum cuts and judicious partitions in graphs without short cycles, J. Combin. Theory Ser. B 88 (2003), 329-346.

[2] R. Aharoni, E.C. Milner and K. Prikry, Unfriendly partitions of a graph, J. Combin. Theory Ser. B 50 (1990), 1-10.

[3] N. Alon, Bipartite subgraphs, Combinatorica 16 (1996), 301-311. 
[4] N. Alon and E. Halperin, Bipartite subgraphs of integer weighted graphs, Discrete Math. 181 (1998), 19-29.

[5] N. Alon, M. Krivelevich and B. Sudakov, MaxCut in $H$-free graphs, in press.

[6] L.D. Andersen, D.D. Grant, and N. Linial, Extremal $k$-colourable subgraphs, Ars Combin 16 (1983), 259-270.

[7] B. Bollobás, A. Gyárfás, J. Lehel and A.D. Scott, Maximum directed cuts in acyclic digraphs, submitted.

[8] B. Bollobás, B. Reed and A. Thomason, An extremal function for the achromatic number, in Graph structure theory (Seattle, WA, 1991) (eds. N. Robertson and P. Seymour), Contemp. Math., 147, Amer. Math. Soc., Providence, RI, USA (1993), pp. 161-165.

[9] B. Bollobás and A.D. Scott, On intersections of graphs, submitted.

[10] B. Bollobás and A.D. Scott, Judicious $k$-partitions, submitted.

[11] B. Bollobás and A.D. Scott, Discrepancy in graphs and hypergraphs, Combinatorica, in press.

[12] B. Bollobás and A.D. Scott, Judicious partitions of bounded-degree graphs, J. Graph Theory 46 (2004), 131-143.

[13] B. Bollobás and A.D. Scott, Problems and results on judicious partitions, Random Structures Algorithms 21 (2002), 414-430.

[14] B. Bollobás and A.D. Scott, Better bounds for Max Cut, in Contemporary combinatorics, Bolyai Soc. Math. Stud., 10, János Bolyai Math. Soc., Budapest (2002), pp. 185-246.

[15] B. Bollobás and A.D. Scott, Judicious partitions of 3-uniform hypergraphs, European J. Combin. 21 (2000), 289-300.

[16] B. Bollobás and A.D. Scott, Exact bounds for judicious partitions of graphs, Combinatorica 19 (1999), 473-486.

[17] B. Bollobás and A.D. Scott, Judicious partitions of hypergraphs, J. Combin. Theory Ser. A 78 (1997), 15-31.

[18] B. Bollobás and A.D. Scott, Judicious partitions of graphs, Period. Math. Hungar. 26 (1993), 125-137.

[19] J.A. Bondy and S.C. Locke, Largest bipartite subgraphs in triangle-free graphs with maximum degree three, J. Graph Theory 10 (1986), 477-504.

[20] M.M. Deza and M. Laurent, Geometry of cuts and metrics, Algorithms and Combinatorics, 15 Springer-Verlag, Berlin (1997). 
[21] C.S. Edwards, An improved lower bound for the number of edges in a largest bipartite subgraph, in Recent advances in graph theory (Proc. Second Czech. Sympos., Prague, 1974) (ed. M. Fiedler), Academia, Prague (1975), pp. 167181.

[22] C.S. Edwards, Some extremal properties of bipartite subgraphs, Canad. J. Math. 25 (1973), 475-485.

[23] P. Erdős, Some recent problems in Combinatorics and Graph Theory, in Combinatorics and Computing (Proc. 26th Southeastern International Conference on Graph Theory, Boca Raton, 1995) Congressus Numerantium, 112, (1995).

[24] P. Erdős, Problems and results in graph theory and combinatorial analysis, in Graph theory and related topics (Proc. Conf., Univ. Waterloo, Waterloo, Ont., 1977) Academic Press, New York-London (1979), pp. 153-163.

[25] P. Erdős, R. Faudree, J. Pach and J. Spencer, How to make a graph bipartite, J. Comb. Theory, Ser. B $\mathbf{4 5}$ (1988), 86-98.

[26] P. Erdős, A. Gyárfás and Y. Kohayakawa, The size of the largest bipartite subgraphs, Discrete Math. 177 (1997), 267-271.

[27] A. Hajnal and E. Szemerédi, Proof of a conjecture of P. Erdős, in Combinatorial theory and its applications II (Proc. Colloq., Balatonfred, 1969) North-Holland, Amsterdam (1970), pp. 601-623.

[28] T. Hofmeister and H. Lefmann, On k-partite subgraphs, Ars Combin. 50 (1998), 303-308.

[29] G. Hopkins and W. Staton, Extremal bipartite subgraphs of cubic triangle-free graphs, J. Graph Theory 6 (1982), 115-121.

[30] A. Johansson, Asymptotic choice number for triangle-free graphs, DIMACS Tech Rep., Rutgers, New Jersey, 91-5, 1996.

[31] M. Laurent, Max-Cut Problem, in Annotated bibliographies in combinatorial optimization (eds. M. Dell'Amico, F. Maffioli and S. Martell), Wiley-Interscience Series in Discrete Mathematics and Optimization, John Wiley and Sons, Ltd., Chichester (1997).

[32] J. Lehel and Zs. Tuza, Triangle-free partial graphs and edge-covering theorems, Discrete Math. 30 (1982), 59-63.

[33] T. Lengauer, Combinatorial algorithms for integrated circuit layout, in Applicable Theory in Computer Science (ed. B.G. Teubner), John Wiley and Sons, Ltd., Chichester (1990).

[34] S.C. Locke, Maximum k-colorable subgraphs, J. Graph Theory 6 (1982), 123132.

[35] M. Molloy and B. Reed, Graph colouring and the probabilistic method, Algorithms and Combinatorics, 23 Springer-Verlag, Berlin (2002). 
[36] N. Văn Ngọc and Zs. Tuza, Linear-time approximation algorithms for the max cut problem, Combin. Probab. Comput. 2 (1993), 201-210.

[37] S. Poljak and D. Turzık, A polynomial algorithm for constructing a large bipartite subgraph, with an application to a satisfiability problem, Canad. J. Math. 34 (1982), 519-524.

[38] S. Poljak and Zs. Tuza, Maximum cuts and large bipartite subgraphs, in Combinatorial optimization (Papers from the DIMACS special year, 1992-1993) (eds. W. Cook, L. Lovász and P. Seymour), DIMACS Ser. Discrete Math. Theoret. Comput. Sci., 20, Amer. Math. Soc., Providence, RI, USA (1995), pp. 181-244.

[39] S. Poljak and Zs. Tuza, Bipartite subgraphs of triangle-free graphs, SIAM J. Discrete Math. 7 (1994), 307-313.

[40] T.D. Porter, Minimal partitions of a graph, Ars Combin. 53 (1999), 181-186.

[41] T.D. Porter, Graph partitions, J. Combin. Math. Combin. Comput. 15 (1994), $111-118$.

[42] T.D. Porter, On a bottleneck bipartition conjecture of Erdős, Combinatorica 12 (1992), 317-321.

[43] T.D. Porter and Bing Yang, Graph partitions II, J. Combin. Math. Combin. Comput. 37 (2001), 149-158.

[44] J.B. Shearer, A note on bipartite subgraphs of triangle-free graphs, Random Structures Algorithms 3 (1992), 223-226.

[45] S. Shelah and E.C. Milner, Graphs with no unfriendly partitions, in A Tribute to Paul Erdős (eds. A. Baker, B. Bollobás \& A. Thomason), CUP, Cambridge (1990), pp. 373-384.

[46] W. Staton, Edge deletions and the chromatic number, Ars Combin. 10 (1980), 103-106 